\title{
Full Optical Properties of Carbonaceous Aerosols by High Energy Monochromated Electron Energy-loss Spectroscopy
}

\author{
Jiangtao Zhu ${ }^{1,2}$, Peter A. Crozier ${ }^{1}$, Toshihiro Aoki ${ }^{2}$ and James R. Anderson ${ }^{1}$ \\ ${ }^{1}$ School for Engineering of Matter, Transport and Energy, Arizona State University, Tempe, AZ, USA, \\ 85287 \\ ${ }^{2}$ LeRoy Erying Center for Solid State Science, Arizona State University, Tempe, AZ, USA, 85287
}

Carbonaceous aerosols have a strong impact on the global climate by direct radiative forcing via light absorption and scattering, and/or indirect radiative forcing via influencing the formation of rain or snow. It is critical to determine their optical properties to understand their contribution to direct radiative forcing. It is also important to understand their local chemical composition which would affect their role in cloud dynamics. By employing monochromated electron energy loss spectroscopy in a newly installed Nion UltraSTEM 100 at ASU with a sub $20 \mathrm{meV}$ energy resolution, it is now possible to determine optical properties down to the infrared range. We can now determine the optical properties of carbonaceous aerosols over the full range of incoming solar radiation 200-2500 $\mathrm{nm}$. In addition, the composition variance in each aerosol spherule are also studied because they are usually assumed to be homogeneous though different types of aerosols particles could be mixed with each other together.

Depending on the sources and the combustion conditions, different types of carbonaceous aerosols [1], such as graphitic black soot, amorphous brown carbon, and humic-like substances, can be formed and present in the atmosphere. Here two types of carbonaceous aerosols, graphitic carbon and amorphous carbon collected from East Asia, were investigated. Low energy loss spectra of these two types of aerosols were collected from Nion UltraSTEM 100 at $60 \mathrm{kV}$ with about $40 \mathrm{meV}$ of full width at half maximum (FWHM) of the zero loss peak (ZLP) and an energy dispersion of $0.01 \mathrm{eV} /$ channel. As shown in Fig. 1a, the EELS of graphitic and amorphous carbons are different, which is related to their different microstructure and chemical composition. Graphitic soot has a much narrower $\pi$ plasmon (4-6 eV) resonance than amorphous carbon. In our previous study, we found that the standard thin film formulation of Kramers-Kronig analysis can be employed to make accurate determination of the dielectric function for carbonaceous particles down to about $40 \mathrm{~nm}$ in size [2]. We applied the same techniques to the set of data collected from Nion UltraSTEM and got the optical properties of carbonaceous aerosols. Figure $1 \mathrm{~b}$ and $\mathrm{c}$ show the complex refractive indices $(n-\mathrm{i} k)$ of black carbon spherules and amorphous carbon spheres in over the photon wavelength range of 200-2500 $\mathrm{nm}$. The absorption coefficient $k$ for both types of carbonaceous aerosols decreases with longer wavelength in the optical spectral range. The absorption in the infrared range is smaller than that in the UV and visible range. In the whole solar radiation range, the absorption coefficient $k$ of graphitic carbon is larger than that of amorphous carbon. The variances in the refractive indices of different particles were related to their variances in composition.

The chemical compositions of carbonaceous aerosols were characterized by energy dispersive X-ray spectroscopy (EDS) on a JEOL ARM 200F. The main element inside both graphitic and amorphous carbon soot is $\mathrm{C}$ which accounts for $90 \mathrm{wt} \%$. We find that on the surface of the graphitic spherule, there is a several nanometer thick layer rich in silicon and oxygen (Fig. 2). The silicon is in the form of silicon dioxide based on their EELS Si $\mathrm{L}_{3,2}$ edge structure. Other trace element, such as $\mathrm{S}, \mathrm{K}, \mathrm{Ca}$, and $\mathrm{Ga}$, appear to be homogeneously distributed through the soot spherule. Similar to the graphitic soot, the 
amorphous carbonaceous aerosols also have a silica coating on their surface (Fig. 3). The nano-size coating could modify the chemical interactions of the carbonaceous aerosols, for example, by changing their ice nucleation properties [3].

\section{References}

[1] J. Zhu, P.A. Crozier, J.R. Anderson, Atmos Chem Phys. 13 (2013), p. 6359.

[2] J. Zhu, P.A. Crozier, P. Ercius, J.R. Anderson, submitted to Microsc Microanal.

[3] The authors acknowledge support of the National Institute of Standards and Technology under Award 60NANB10D022. The authors acknowledge the use of facilities in John M. Cowley Center for High Resolution Electron Microscopy at Arizona State University.
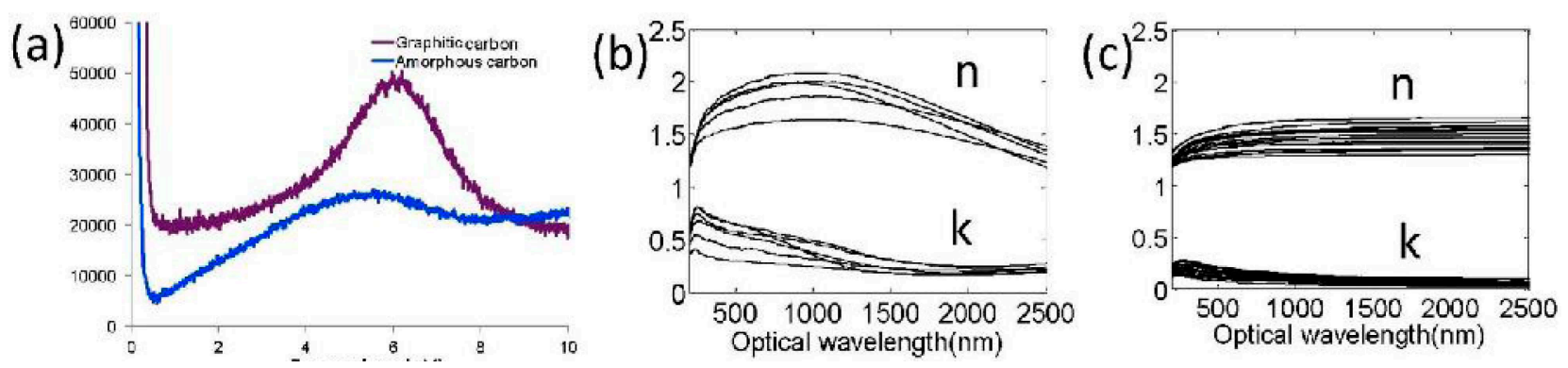

Figure 1. (a) Low loss EELS of graphitic and amorphous carbon. Refractive index (n-ik) in the range of 200-2500 nm in optical wavelength of (b) graphitic carbon and (c) amorphous carbon.
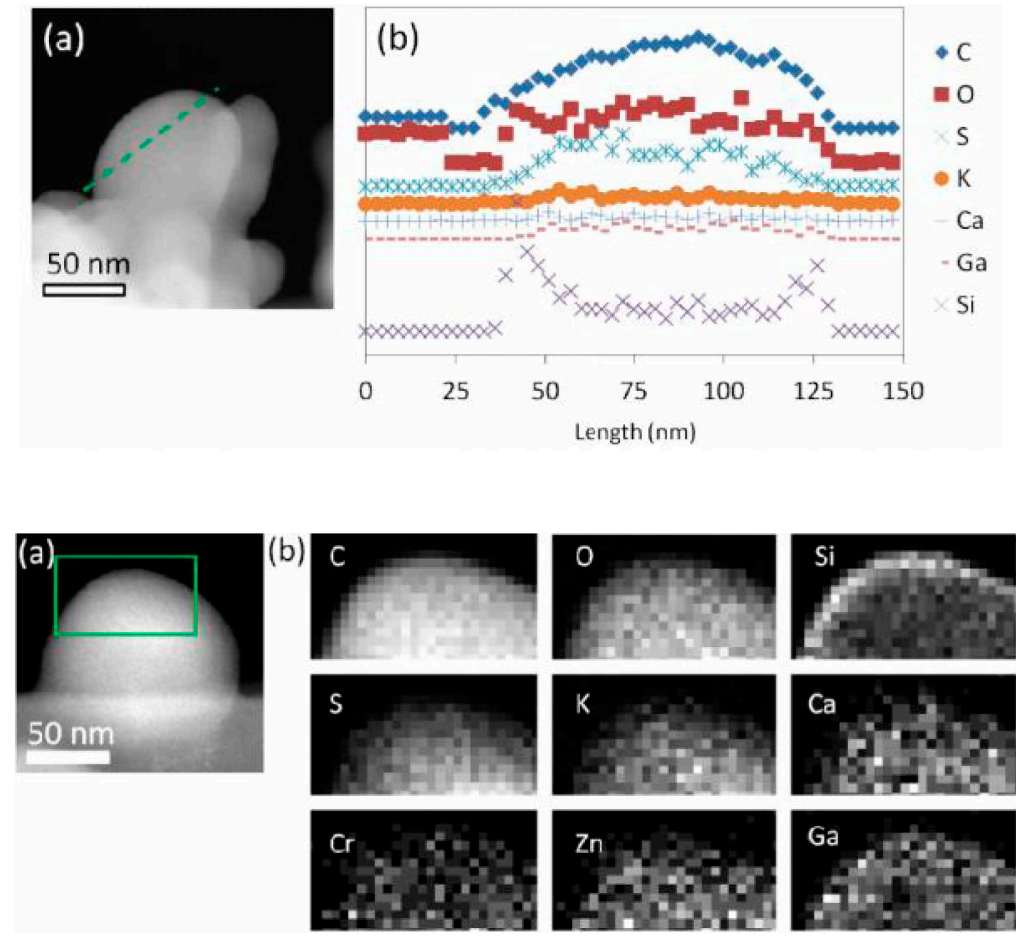

Figure 2. Element distributions of a typical graphitic carbonaceous aerosol by EDS. (a) The angular dark field survey image. (b) Intensity distributions of element $\mathrm{C}, \mathrm{O}, \mathrm{S}, \mathrm{K}$, $\mathrm{Ca}, \mathrm{Ga}$ and $\mathrm{Si}$ along the line in the image (a).
Figure 3. Element mapping of a typical amorphous carbonaceous aerosol by EDS: (a) the angular dark field survey image, (b) intensity distributions of different elements: C, O, S, Si, K, Ca, Zn and $\mathrm{Ga}$ of the area in image (a). 